\title{
Neonatal brachial plexus palsy: From conservative management to nerve reconstruction
}

\author{
K A Nihal Gunatillaka ${ }^{1}$ \\ Sri Lanka Journal of Child Health, 2005; 34: 52-5
}

(Key words: brachial plexus palsy, birth injuries )

\section{Introduction}

Brachial plexus injuries are uncommon complications of childbirth. Incidence is about 1 in 1000 births and is rising due to increase in birth weights ${ }^{1}$. Awareness of this condition is increasing due to resurgence of operative intervention and the rise in litigation.

\section{Aetiology}

Factors associated with brachial plexus palsy are'2:

- $\quad$ Large birth weight $(>3.5 \mathrm{~kg})$

- Multiparous mother

- Shoulder dystocia

- Prolonged second stage

- Assisted delivery - vacuum or forceps

Elective caesarean section provides some protection but does not completely eliminate the risk of brachial plexus injury ${ }^{3}$.

\section{The pattern and degree of nerve injury}

The anatomical arrangement and structure of the plexus (Figure 1$)^{4}$ explains the pattern of injuries that are usually seen. With an increasing angle between the head and the shoulder, tension increases on the brachial plexus. The tension is greatest in the uppermost roots or trunks and gradually diminishes towards the middle and lower trunks and roots. Fifth

${ }^{1}$ Consultant Rheumatologist, Department of
Rheumatology, Lady Ridgeway Hospital, Colombo. cervical (C5) nerve root has the strongest dural fibrous attachment and hence avulsion of the nerve root from the anterior intervertebral foramen is uncommon. Instead, the tension causes a rupture at the next most vulnerable spot i.e. at the commencement of the upper trunk. The sixth and seventh cervical roots, by comparison, are not firmly attached and are more likely to be avulsed. The eighth cervical and first thoracic roots are not in the direct line of pull and are only usually injured once the tension has ruptured or avulsed the upper root/trunks thus allowing the tension to be taken up in these lower nerves. The exception is in Klumpke palsy where the injury is caused by hyper-abduction of the arm as may occur in a breech delivery. Depending on the degree of force, its suddenness of application and the susceptibility of the nerve, different types of injuries may result. Neuropraxia results from disruption of the myelin sheath producing a conduction block. Disruption of some or all of the axons with preservation of the more resistant endo-peri-epineurium sheath is called axonotmesis. In neurotmesis there is a complete rupture of the nerve i.e. axons and various sheaths, so that there is no connection between the proximal and distal ends. Neuropraxia is fully reversible as soon as the myelin sheath is regenerated. Axonotmesis has a chance of regeneration as the preserved sheath guides the regenerating axons to their original destination. However, in neurotmesis, the regenerating axons cannot find their way to their end points and instead form a neuroma. 


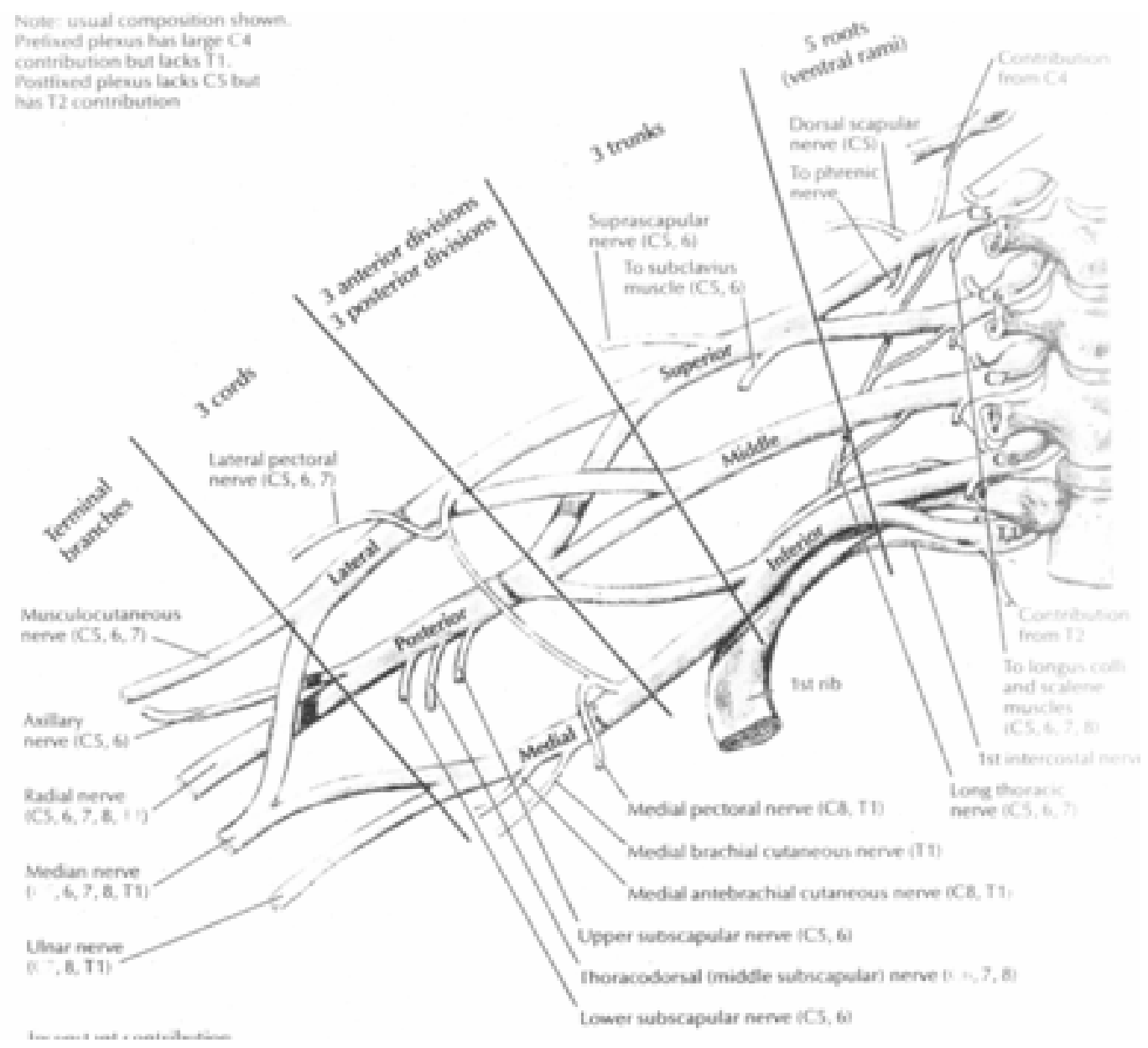

Figure 14 Anatomical arrangement of brachial plexus

\section{Natural history}

Surveys of neonatal cases in obstetric units report spontaneous recovery rates ranging from $75-95 \%{ }^{5}$. Co-existing Horner syndrome, diaphragmatic paralysis and flaccid paralysis of entire limb are adverse prognostic features. The challenge lies in the management of this 5-25\% of infants who are destined for poor functional outcome. Neurosurgical reconstruction can be beneficial to this group. However, to achieve optimal results, surgery needs to be performed within the first year of life ${ }^{2}$. At this age, the capacity for nerve regeneration and cortical reintegration is high. More importantly, muscle reinnervation can occur before motor end plate drop off becomes irreversible ${ }^{2}$.

Bennet and Harold ${ }^{6}$ found that all their patients who achieved complete recovery had begun to show improvement by 2 weeks of age. The Collaborative Perinatal Study $^{7}$ indicated that $93 \%$ of patients destined to attain full recovery had done so by 4 months. In a seminal, long term study of 44 conservatively managed infants, Gilbert and Tassin ${ }^{8}$ found that all patients who made satisfactory recovery had detectable deltoid and bicep contractions by 3 months. More precisely, patients who proceed to complete recovery have antigravity strength in deltoid and biceps by end of second month and in external rotators by end of the third month. Patients still make a satisfactory, though imperfect, recovery if there are perceptible contractions in biceps and deltoid by end of third month and progress to develop antigravity strength by end of fifth month. Similarly, Metaizeau et $\mathrm{al}^{9}$ found that patients who exhibit no sign of recovery by 3 months ultimately have unsatisfactory functional outcomes. With regard to individual muscle groups, they asserted that if there is no progress towards recovery between 3 and 6 months, ultimate likelihood of further spontaneous improvement in the muscle group in question is essentially nil. 
Most surgeons currently reporting experiences with plexus reconstruction believe that the decision for reconstruction can be made reliably between $3 \mathrm{rd}$ and 6th month. Spontaneous recovery is complete by 12 to 18 months.

\section{Investigations}

Investigations have been found to be disappointing in both diagnosis of the degree of nerve injury and in localization of injury. MRI, CT, CT myelography, myelography and electrophysiological studies show little correlation with surgical findings and eventual outcome.

\section{Diagnosis}

Once a clinical diagnosis is made a chest radiograph is arranged to exclude phrenic nerve palsy (raised hemidiaphragm) and clavicular / humeral fractures. Presence or absence of Horner syndrome should be documented clearly as this has implications for future management and prognosis.

\section{Management}

No immediate management is indicated other than informing parents to avoid pulling on affected limb to lift the child and to protect the limb from dragging behind when moving the child. Splinting with shoulder in abduction and upper arm in external rotation in the neonatal period is NOT beneficial and could lead to excessive mobility of the glenohumeral joint and dislocation. The child should be reassessed at six weeks and particular note should be made of shoulder abduction (deltoid C5), elbow flexion (biceps C6), elbow and wrist extension (triceps and extensor mass $\mathrm{C} 7$ ) and finger flexion (flexor digitorium profundus $\mathrm{C} 8$ ).

\section{Therapy}

Whilst awaiting recovery to occur spontaneously it is essential to maintain suppleness in the joints and muscles. Joint stiffness or poor range of motion can frustrate efforts made by regenerating muscles to move the involved joint and hence impede potential recovery. Regenerating muscle often fails to reach pre-injury power levels, and their effectiveness may be further reduced by joint and muscle stiffness. Joint suppleness is maintained by daily exercises through the full passive range of motion. These rang of motion exercises involving the shoulder should be deferred for 3-4 weeks to allow the painful injuries to heal and to eliminate the risk of bleeding into the sheath, resulting in reduced spontaneous recovery rates. The range of motion exercises should be performed with every nappy change and bath. They should also be performed with both arms simultaneously to stabilize the trunk and scapulae. Positioning can also be used to minimize the contractures and maintain joint suppleness. Characteristic positions which are helpful would be:

- Supine with upper limbs placed close to the trunk with back of the hand in contact with bed.

- Supine with hands at back of the head.

- Supine with hands at the back.

- Supine with hands placed anteriorly over the opposite shoulder.

- $\quad$ Supine with both shoulders abducted to $90^{\circ}$ and arms externally rotated - "hands up, you are under arrest" position

Stimulation, whether tactile or electrical, will encourage the infant to attend to the paretic limb. This would be helpful in the long term to minimize neglect / learned non-use of the affected limb. Electrical stimulation, in particular, may prevent muscle atrophy or increase the muscle bulk. However, this does not accelerate nerve healing ${ }^{1}$ or improve long term outcome. Stimulation can also be used to activate paralyzed muscles, initially in isolation but later with recovery, in functional activities. Carefully planned play therapy, sand play, bimanual activities and constraint induced therapy can all be used successfully in the rehabilitation of the older child. Functional splints to hold thumb in some abduction and the wrist in extension may also force weak muscles to contract. This can be used to strengthen selected muscle groups if used with appropriate functional / play activities.

\section{Reconstruction surgery}

Following dismal attempts at surgical repair in the 1920 s it had been accepted that surgery had little to offer these patients. However, advent of microsurgery, improvement in nerve repair, understanding of nerve physiology, and importantly improvement in child anaesthesia, has changed that situation. Gilbert and his associates1 in Paris deserve credit for reviving interest in reconstructive surgery using modern microsurgical techniques for birth injuries of the brachial plexus in 1980s. 


\section{Indications}

Despite lack of randomized controlled trials, available evidence suggests that surgical exploration and repair leads to a better result and is thus indicated in: ${ }^{2}$

- Complete plexus palsies and a Horner syndrome / phrenic nerve palsy / spinal cord injury.

- C5, C6 lesions with evidence of phrenic nerve palsy.

- Babies who fail to recover any biceps contraction and wrist extension by end of 3 months. These babies can be reassessed when they attend for surgery at 4-6 months of age and if any significant recovery is seen the decision for surgery can be altered.

Atrophy of muscles sets an upper limit for the time of operation. There is probably little benefit from plexus exploration after 18 months of age.

Repair is either by direct nerve repair, repair with use of a nerve graft (sural nerve) or in the case where proximal end of nerve does not exist (due to avulsion) the distal nerve is neurotized by connecting it to another nerve, either within plexus (intra-plexural) or external to the plexus (extraplexural). The accessory or intercostal nerves are used for this purpose.

Following a 3 week period of immobilization in a sling to protect the reconstruction, therapy is restarted. Nerve regeneration takes many months and the recovery in the shoulder takes at least nine and the hand at least eighteen months.

Even after the most successful plexus reconstruction, normal strength is not restored to every muscle group, and varying degrees of joint capsule or muscle contractrures or bony deformities are not uncommon. Joint capsule release, release of muscle contractures, tendon transfers or osteotomies can be used to rectify such functional limitations.

\section{Conclusion}

Brachial plexus palsy is an uncommon condition. The majority resolves spontaneously with minimal or no disability. Management of the remaining patients (approximately 10\%) is complex and prolonged. These infants have substantial disability with difficulty in reaching, using the affected hand and impairment of bimanual function. Prognosis depends principally on extent of the injury. However the potential for recovery is also influenced by the development of learned non use (neglect) of the limb and soft tissue contractures. Management involves regular assessment, patient and family support, planned physio- and occupational therapy, timely neurosurgical reconstruction in carefully selected infants and orthopaedic techniques to optimize returned function.

\section{References}

1. Piatt J H. Birth injuries of the brachial plexus. Paediatric Clinics of North America 2004; 51: 421-40.

2. Giele H. Management of obstetrical brachial plexus palsy. Current Paediatrics 1999; 9: 1827.

3. Graham E M, Forouzan J, Morgan M A. A retrospective analysis of Erb Palsy cases and their relation to birth weight and trauma at delivery. Journal of Maternal and Fetal Medicine 1997; 6 (1): 1-5.

4. Netter F H. editor, Atlas of orthopaedic anatomy. Basle, Switzerland: Ciba-Geigy Ltd, 1989; Plate 31.

5. Noetzel M J, Park T S, Robinson S, Kaufman B. Prospective study of recovery following neonatal brachial plexus injury. Journal of Child Neurology 2001; 16 (7): 488-92.

6. Bennet G C, Harold A J. Prognosis and Early Management of birth injuries to the brachial plexus. BMJ 1976; 1 (6024); 1520-1.

7. Gordon M, Rich H, Deuschberger J, Green M. The immediate and long term outcome of obstetric birth trauma. American Journal of Obstetrics and Gynaecology 1973; 117 (1):51-6.

8. Gilbert A, Tassin J L. Obstetrical palsy: A clinical, pathologic and surgical review. In Terzis J K, editor. Micro reconstruction of nerve injuries. Philadelphia: W B Saunders, 1987; 529-53.

9. Metaizeau J P, Prevot J, Lascombes P. Obstetrical paralysis. Spontaneous development and results of early micro surgical treatment. Annals of Paediatrics (Paris) 1984; 31 (2): 93102. 
